\title{
The Influence of Occlusion Time on Measuring Respiratory Resistance and Compliance in Infants with Bronchiolitis
}

\author{
B. STEINBRUGGER, J. FABIAN, AND M. S. ZACH
}

Department of Pediatrics, University of Graz, Austria

\begin{abstract}
The passive, single-breath, flow-volume technique is a simple method for measuring the resistance (Rrs) and the compliance (Crs) of the respiratory system in infants. So far, the potential influence of end inspiratory occlusion time on these measurements has not been investigated. We measured Rrs and Crs in 36 infants and toddlers with bronchiolitis; in each child, a spectrum of nine fixed occlusion times, ranging from 90 to $600 \mathrm{~ms}$, was applied in random sequence. Increasing the duration of occlusions from 90 to $275 \mathrm{~ms}$ resulted in marked stepwise changes of measured Rrs and Crs; occlusions longer than $275 \mathrm{~ms}$, however, produced highly reproducible measurements, as expressed 1) by minimal absolute differences between measured values at subsequent occlusion times and 2) by minimal percentage changes of measured values from one occlusion time to the next. There was no influence of age on the results; reproducible measurements were made in children as old as $1.5 \mathrm{y}$. This suggests that, in infants with bronchiolitis, 1 ) occlusions between 300 and $450 \mathrm{~ms}$ might be ideal for obtaining reliable measurements, and 2) the age range for applying this method can be extended into the 2nd y of life. (Pediatr Res 33: 273-277, 1993)
\end{abstract}

Abbreviations

VE, expired volume

$\dot{\mathrm{VE}}$, expiratory flow

Pao, pressure measured at airway opening

Rrs, resistance of the respiratory system

Crs, compliance of the respiratory system

Trs, time constant of the respiratory system

More than three decades ago, Comroe et al. (1) described a technique for measuring respiratory mechanics in animals by a single passive deflation. Subsequently, McIlroy et al. (2) plotted $\dot{V E}$ against $V E$ to obtain the time constant of this passive exhalation in anesthetized human subjects. Mortola et al. (3), as well as LeSouef et al. $(4,5)$, then adapted this "single-breath technique" for measuring passive respiratory mechanics in newborns and infants; in this age group, relaxation of the respiratory muscles is achieved by activating the Hering-Breuer reflex via an end-inspiratory occlusion of the airway. When the Pao reaches a plateau, muscle relaxation and pressure equilibration have occurred. The subsequent termination of the occlusion results in a

Received March 23, 1992; accepted September 22, 1992

Correspondence and reprint requests: Dr. Bernhard Steinbrugger, Pediatric Department, Respiratory and Allergic Disease Division, Univ.-Kinderklinik, Auenbruggerplatz, A-8036 Graz, Austria.

Supported by grant no. 3228 of the Jubiläumsfonds der Österreichischen Nationalbank. passive exhalation, which is recorded in a flow-volume mode. From the linear portion of this plot, a Trs is calculated. Trs together with Pao allows for calculating the Rrs and Crs.

This simple technique might develop into a routine method for assessing lung function in infants; as a prerequisite, however, several methodologic questions must be addressed. One problem that so far has not been sufficiently evaluated is the question of "optimum end-inspiratory occlusion time." Too-short occlusions do not result in complete muscle relaxation and pressure equilibration. As is evident from the lack of any linear portion of the expiratory flow-volume plot, too-long ones are disturbed by recommencing respiratory muscle activity.

So far, most investigators have observed Pao visually and have released their manual occlusion when the tracing appeared flat. A true pressure plateau should be characterized by a zero change over a defined period of time; as yet, however, no relevant standardization efforts have been made. In preliminary trials, we encountered several practical difficulties when trying to focus on the pressure plateau for a timely termination of the occlusion. In some children, one could observe two plateaus, separated by a small pressure step; in others, the tracing, while appearing flat, continued to show further small changes of Pao on closer inspection. Frequently, manual occlusions are released too late, resulting in tracings that are distorted by recommencing respiratory muscle activity.

Consequently, we decided to evaluate the question of "optimum occlusion time" without focusing on the pressure plateau; our indirect approach was based on applying a series of different occlusion times to each patient. We thereby addressed the following questions: 1) Does the duration of the occlusion have an influence on the measurement of Pao, Rrs, and Crs? 2) If so, is there a range of occlusion times over which the measured values of Pao, Rrs, and Crs do not differ significantly? Reproducibility of measured values over such an occlusion time range would suggest a satisfactory degree of respiratory muscle relaxation and pressure equilibration and, thereby, would indicate optimum end-inspiratory occlusion time.

\section{MATERIALS AND METHODS}

Forty infants and toddlers, referred for lung function testing because of acute viral bronchiolitis, were recruited for the study. Two infants with imminent respiratory failure were excluded. Also excluded were two other infants on the basis of repeatedly alinear flow-volume tracings. The remaining 36 patients studied, 23 boys and 13 girls, covered an age range from 3 to $79 \mathrm{wk}$; anthropometric data and respiratory rates are given in Table 1 . The children were studied between the $3 \mathrm{rd}$ and the $7 \mathrm{th} \mathrm{d}$ of their hospitalization. Clinically, their airway disease ranged from mild to moderate. On the basis of age, this patient group was divided into three subgroups: subgroup A consisted of 13 infants aged less than $6 \mathrm{mo}$; subgroup B, 11 infants aged from 6 to $9 \mathrm{mo}$; and subgroup C, 12 infants and toddlers older than 9 mo. 
Table 1. Anthropometric data*

\begin{tabular}{|c|c|c|c|c|}
\hline & $\begin{array}{c}\text { Total } \\
(3-79 w k)\end{array}$ & $\begin{array}{c}\text { Subgroup A } \\
(<26 \text { wk })\end{array}$ & $\begin{array}{c}\text { Subgroup B } \\
(27-38 \text { wk) }\end{array}$ & $\begin{array}{c}\text { Subgroup C } \\
(>39 \text { wk) }\end{array}$ \\
\hline No. of patients & 36 & 13 & 11 & 12 \\
\hline Age (wk) & $33.0 \pm 16.7$ & $17.7 \pm 6.2$ & $30.1 \pm 3.0$ & $52.3 \pm 12.3$ \\
\hline Weight (kg) & $7.9 \pm 2.2$ & $6.1 \pm 1.9$ & $8.0 \pm 1.5$ & $9.9 \pm 0.9$ \\
\hline Length $(\mathrm{cm})$ & $68.3 \pm 7.4$ & $62.5 \pm 6.1$ & $67.7 \pm 3.5$ & $75.8 \pm 4.7$ \\
\hline Respiratory rate & $36.8 \pm 7.8$ & $39.0 \pm 6.6$ & $39.7 \pm 7.7$ & $31.8 \pm 7.1$ \\
\hline
\end{tabular}

* Mean \pm 1 SD.

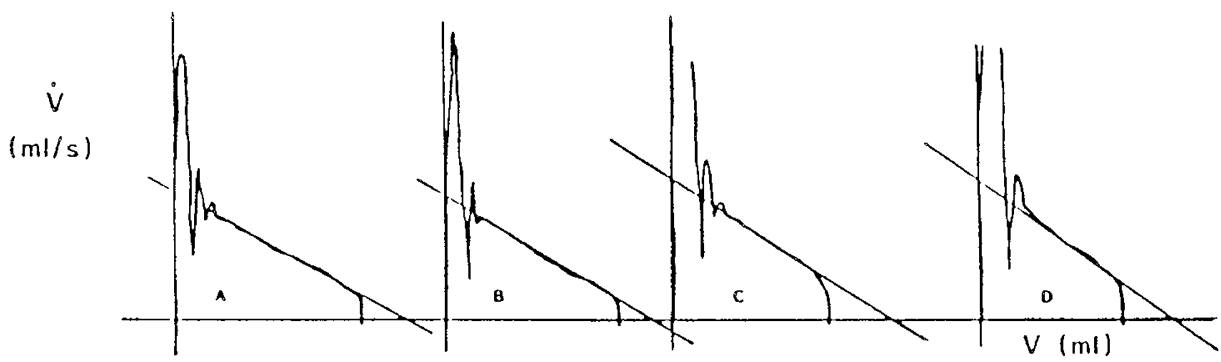

Fig. 1. VE-VE curves at different occlusion times, as obtained in a boy $46 \mathrm{wk}$ of age with acute bronchiolitis. Also shown is the line drawn manually through the descending straight part of the $\dot{V E} / \mathrm{VE}$ plot. $\dot{\mathrm{V}}$, expiratory flow; $\mathrm{V}$, expired volume. $A$, occlusion time $=90 \mathrm{~ms} ; B$, occlusion time $=215 \mathrm{~ms} ; C$, occlusion time $=320 \mathrm{~ms} ;$ and $D$, occlusion time $=520 \mathrm{msec}$.

Table 2. Measurements at different occlusion times*

\begin{tabular}{ccccccc}
\hline $\begin{array}{c}\text { Occlusion time } \\
(\mathrm{ms})\end{array}$ & $\mathrm{VE}(\mathrm{mL})$ & $\begin{array}{c}\dot{\mathrm{VE}}(\mathrm{mL} / \mathrm{s}) \\
\mathrm{PaO}(\mathrm{cm}\end{array}$ & $\begin{array}{c}\mathrm{Rrs}\left(\mathrm{cm} \mathrm{H} \mathrm{H}_{2} \mathrm{O} /\right. \\
\mathrm{mL} / \mathrm{s})\end{array}$ & \multicolumn{2}{c}{$\mathrm{CRS}(\mathrm{mL} / \mathrm{cm}$} \\
$\left.\mathrm{H}_{2} \mathrm{O}\right)$ & $\mathrm{Trs}(\mathrm{s})$ \\
\hline 90 & $160 \pm 56$ & $199 \pm 62$ & $5.4 \pm 2.2$ & $0.028 \pm 0.012$ & $34.8 \pm 18.9$ & $0.832 \pm 0.362$ \\
140 & $154 \pm 57$ & $206 \pm 68$ & $8.4 \pm 2.4$ & $0.042 \pm 0.015$ & $20.2 \pm 8.8$ & $0.763 \pm 0.275$ \\
215 & $157 \pm 53$ & $220 \pm 63$ & $9.9 \pm 2.5$ & $0.047 \pm 0.016$ & $16.7 \pm 7.3$ & $0.718 \pm 0.232$ \\
275 & $155 \pm 50$ & $224 \pm 68$ & $10.5 \pm 2.6$ & $0.050 \pm 0.017$ & $15.6 \pm 6.6$ & $0.716 \pm 0.266$ \\
320 & $152 \pm 49$ & $230 \pm 68$ & $10.9 \pm 2.7$ & $0.051 \pm 0.018$ & $14.8 \pm 6.2$ & $0.686 \pm 0.252$ \\
380 & $153 \pm 50$ & $233 \pm 67$ & $11.3 \pm 2.9$ & $0.052 \pm 0.019$ & $14.2 \pm 6.1$ & $0.681 \pm 0.261$ \\
450 & $150 \pm 47$ & $235 \pm 67$ & $11.7 \pm 2.8$ & $0.054 \pm 0.019$ & $13.5 \pm 5.4$ & $0.663 \pm 0.238$ \\
520 & $141 \pm 43$ & $236 \pm 72$ & $12.5 \pm 3.5$ & $0.057 \pm 0.021$ & $12.2 \pm 5.4$ & $0.633 \pm 0.231$ \\
600 & $148 \pm 45$ & $225 \pm 57$ & $12.8 \pm 4.3$ & $0.061 \pm 0.021$ & $12.4 \pm 6.6$ & $0.691 \pm 0.270$ \\
\hline
\end{tabular}

$*$ Mean $\pm 1 \mathrm{SD}$.

Table 3. Intraindividual variability (coefficients of variation)

\begin{tabular}{ccccc}
\hline $\begin{array}{c}\text { Occlusion time } \\
(\mathrm{ms})\end{array}$ & Pao (\%) & Rrs (\%) & Crs (\%) & Trs (\%) \\
\hline 90 & $11.9 \pm 6.2^{*}$ & $12.7 \pm 8.1$ & $11.6 \pm 5.3$ & $11.0 \pm 5.6$ \\
140 & $7.4 \pm 4.6$ & $8.6 \pm 5.1$ & $7.6 \pm 5.5$ & $10.0 \pm 4.8$ \\
215 & $6.6 \pm 4.4$ & $6.9 \pm 3.1$ & $7.0 \pm 3.4$ & $10.4 \pm 4.6$ \\
275 & $6.0 \pm 3.3$ & $7.0 \pm 4.1$ & $6.4 \pm 3.2$ & $9.4 \pm 4.7$ \\
320 & $5.0 \pm 2.5$ & $5.4 \pm 3.3$ & $5.4 \pm 3.0$ & $8.2 \pm 4.0$ \\
380 & $5.7 \pm 4.0$ & $5.8 \pm 3.9$ & $6.0 \pm 3.9$ & $8.6 \pm 6.7$ \\
450 & $4.8 \pm 3.1$ & $6.1 \pm 3.3$ & $5.6 \pm 2.7$ & $9.2 \pm 4.1$ \\
520 & $6.0 \pm 4.0$ & $4.6 \pm 2.6$ & $6.5 \pm 4.7$ & $8.8 \pm 5.9$ \\
600 & $4.9 \pm 2.9$ & $5.2 \pm 2.4$ & $5.4 \pm 1.7$ & $7.2 \pm 3.9$ \\
\hline
\end{tabular}

* Mean \pm 1 SD.

Informed consent was obtained from the parents. One or both of the parents were present during the investigation. Before lung function testing, each infant fasted for $3 \mathrm{~h}$, then was sedated with orally administered chloralhydrate $(60$ to $80 \mathrm{mg} / \mathrm{kg}$ body weight), and subsequently was fed. Lung function was measured with the infant sleeping in the supine position.

Passive respiratory mechanics, specifically Rrs and Crs, were measured by the single-breath occlusion technique, as described elsewhere $(4,5)$. Briefly, end-inspiratory airway occlusion stimulates the Hering-Breuer reflex, thereby inducing relaxation of the respiratory muscles against the shutter. Under these condi- tions, Pao equals the passive recoil pressure of the respiratory system. The subsequent termination of the occlusion results in a relaxed expiration; its linear portion is extrapolated to the volume- and flow-axis of the flow-volume plot. These volume and flow measurements, together with Pao, allow for calculating Rrs and Crs.

In each infant, nine fixed end-inspiratory occlusion times $(90$, $140,215,275,320,380,450,520$, and $600 \mathrm{~ms}$ ) were applied in a randomized sequence. Several end-inspiratory occlusions were performed with each occlusion time; for measuring Pao, passively expired volume, and $\dot{V E}$, the mean values of four to eight 

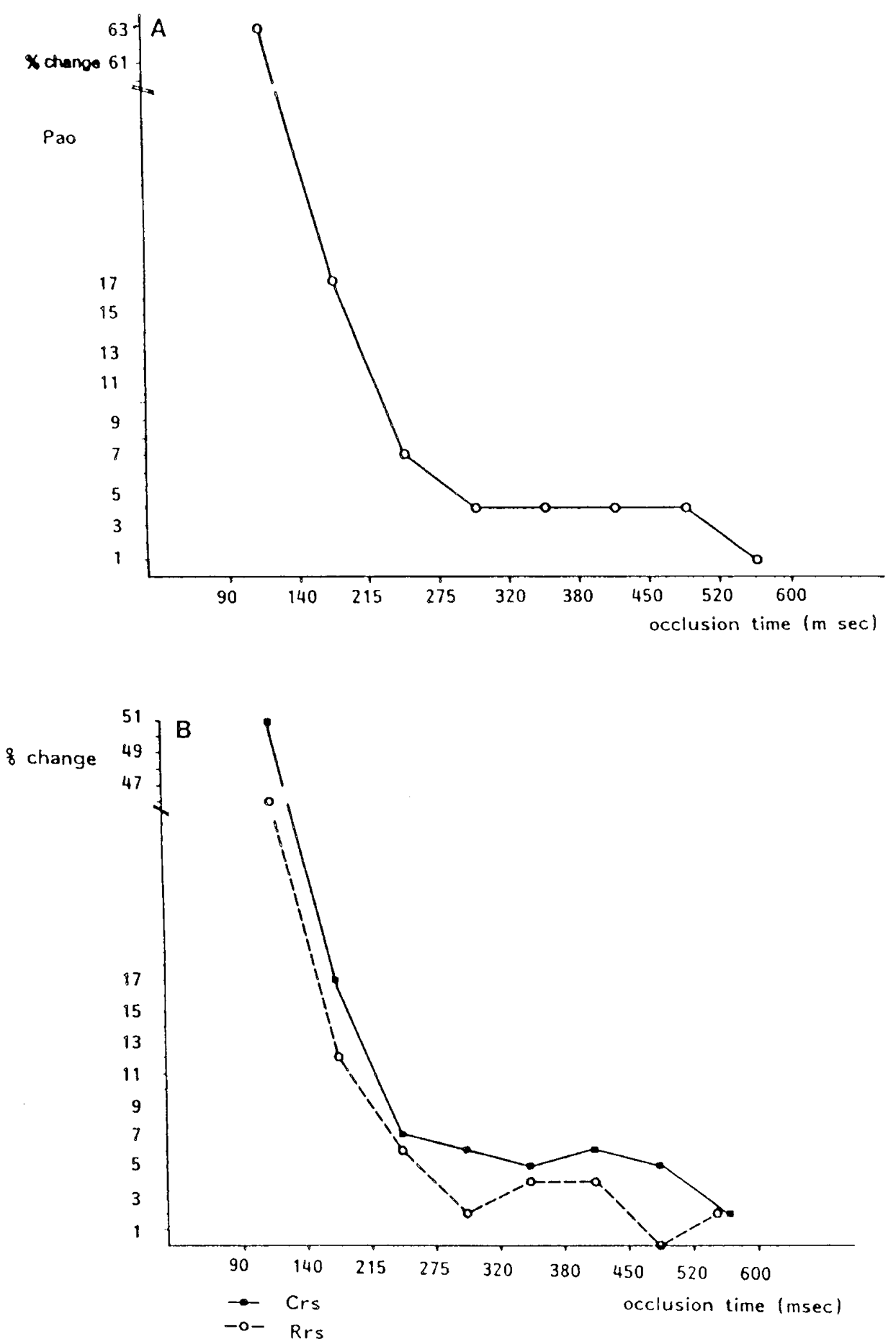

Fig. 2. A, Mean percentage change of measured Pao from one occlusion time to the next. $B$, Mean percentage change of measured Rrs and Crs from one occlusion time to the next.

satisfactory curves were used. In accordance with previous work $(2,6)$, a straight linear relationship of VE to VE between at least 38 and $75 \%$ of VE was used for defining a satisfactory curve.

The measuring equipment was custom-built for this study. An appropriately sized face mask was connected to the airway opening of the sleeping infant by sealing it with a ring of silicon therapeutic putty around mouth and nose. Connected to the face mask was an electromechanically operated and electronically controlled shutter system with a response time of $20 \mathrm{~ms}$ (Biegler, Mauerbach, Austria). Flow was measured with a Fleisch 0 or 1 pneumotachygraph, attached to a low-pressure-range HBM PD1 (0.01 bar $\left.=10.2 \mathrm{~cm} \mathrm{H}_{2} \mathrm{O}\right)$ differential pressure transducer and an HBM MVD 2406A amplifier (Hottinger Baldwin Messtech- nik, Darmstadt, Germany). The pneumotachygraph was attached distal to the shutter system and thus was not pressurized during occlusions. The obtained flow signal was electronically integrated into volume. Pao was registered using an HBM PDI $\left(0.1 \mathrm{bar}=102 \mathrm{~cm} \mathrm{H}_{2} \mathrm{O}\right)$ pressure transducer with an HBM MVD 2406A amplifier (Hottinger Baldwin Messtechnik). Flow, volume, and pressure signals were recorded in real time on an ATpersonal computer with a WFS-8 sampling chart, with a sampling frequency of $300 \mathrm{~Hz}$ (Biosys, Vienna, Austria).

For statistical evaluation, measurements at different occlusion times were compared by analysis of variance. In addition, the percentage changes of measured values from any given occlusion 
Table 4. Measurements at different occlusion times in subgroup $A^{*}$

\begin{tabular}{|c|c|c|c|c|c|c|}
\hline Occlusion time (ms) & $\mathrm{VE}(\mathrm{mL})$ & $\begin{array}{c}\dot{\mathrm{VE}} \\
(\mathrm{mL} / \mathrm{s})\end{array}$ & $\begin{array}{c}\mathrm{Pao}(\mathrm{cm} \\
\left.\mathrm{H}_{2} \mathrm{O}\right)\end{array}$ & $\begin{array}{c}\mathrm{Rrs}\left(\mathrm{cm} \mathrm{H}_{2} \mathrm{O} /\right. \\
\mathrm{mL} / \mathrm{s})\end{array}$ & $\begin{array}{c}\mathrm{Crs}(\mathrm{mL} / \mathrm{cm} \\
\left.\mathrm{H}_{2} \mathrm{O}\right)\end{array}$ & Trs (s) \\
\hline 90 & $110 \pm 39$ & $166 \pm 45$ & $4.9 \pm 1.9$ & $0.031 \pm 0.013$ & $24.8 \pm 9.7$ & $0.668 \pm 0.215$ \\
\hline 140 & $111 \pm 38$ & $180 \pm 48$ & $8.0 \pm 1.9$ & $0.046 \pm 0.012$ & $14.2 \pm 4.8$ & $0.627 \pm 0.215$ \\
\hline 215 & $110 \pm 36$ & $185 \pm 44$ & $9.5 \pm 1.4$ & $0.054 \pm 0.014$ & $11.5 \pm 3.8$ & $0.605 \pm 0.201$ \\
\hline 275 & $110 \pm 39$ & $196 \pm 58$ & $10.2 \pm 1.7$ & $0.056 \pm 0.016$ & $10.9 \pm 4.1$ & $0.578 \pm 0.206$ \\
\hline 320 & $108 \pm 37$ & $202 \pm 57$ & $10.6 \pm 1.8$ & $0.056 \pm 0.016$ & $10.2 \pm 3.7$ & $0.553 \pm 0.211$ \\
\hline 380 & $108 \pm 39$ & $202 \pm 65$ & $10.9 \pm 1.9$ & $0.058 \pm 0.018$ & $10.0 \pm 3.8$ & $0.557 \pm 0.210$ \\
\hline 450 & $109 \pm 37$ & $206 \pm 63$ & $11.5 \pm 1.4$ & $0.060 \pm 0.018$ & $9.6 \pm 3.8$ & $0.555 \pm 0.220$ \\
\hline 520 & $111 \pm 34$ & $195 \pm 60$ & $12.1 \pm 2.1$ & $0.066 \pm 0.020$ & $9.4 \pm 3.4$ & $0.595 \pm 0.204$ \\
\hline 600 & $102 \pm 41$ & $180 \pm 40$ & $12.5 \pm 3.3$ & $0.071 \pm 0.020$ & $8.4 \pm 4.0$ & $0.653 \pm 0.200$ \\
\hline
\end{tabular}

* Mean $\pm 1 \mathrm{SD}$.

Table 5. Measurements at different occlusion times in subgroup $B^{*}$

\begin{tabular}{|c|c|c|c|c|c|c|}
\hline Occlusion time (ms) & $\mathrm{VE}(\mathrm{mL})$ & $\begin{array}{c}\dot{\mathrm{VE}} \\
(\mathrm{mL} / \mathrm{s})\end{array}$ & $\begin{array}{c}\mathrm{Pao}(\mathrm{cm} \\
\left.\mathrm{H}_{2} \mathrm{O}\right)\end{array}$ & $\begin{array}{c}\operatorname{Rrs}\left(\mathrm{cm} \mathrm{H}_{2} \mathrm{O} /\right. \\
\mathrm{mL} / \mathrm{s})\end{array}$ & $\begin{array}{c}\text { Crs }(\mathrm{mL} / \mathrm{cm} \\
\left.\mathrm{H}_{2} \mathrm{O}\right)\end{array}$ & $\operatorname{Trs}(\mathrm{s})$ \\
\hline 90 & $159 \pm 26$ & $220 \pm 49$ & $6.8 \pm 2.7$ & $0.031 \pm 0.013$ & $28.6 \pm 14.9$ & $0.737 \pm 0.169$ \\
\hline 140 & $156 \pm 28$ & $226 \pm 50$ & $10.1 \pm 3.2$ & $0.046 \pm 0.019$ & $17.4 \pm 6.7$ & $0.703 \pm 0.136$ \\
\hline 215 & $156 \pm 28$ & $233 \pm 43$ & $11.2 \pm 3.6$ & $0.050 \pm 0.018$ & $14.9 \pm 5.2$ & $0.670 \pm 0.088$ \\
\hline 275 & $158 \pm 30$ & $235 \pm 50$ & $12.3 \pm 3.5$ & $0.054 \pm 0.019$ & $13.9 \pm 4.4$ & $0.682 \pm 0.137$ \\
\hline 320 & $151 \pm 27$ & $234 \pm 49$ & $12.6 \pm 3.7$ & $0.056 \pm 0.022$ & $13.0 \pm 3.9$ & $0.657 \pm 0.108$ \\
\hline 380 & $151 \pm 28$ & $246 \pm 45$ & $13.2 \pm 3.9$ & $0.056 \pm 0.022$ & $12.3 \pm 3.7$ & $0.618 \pm 0.100$ \\
\hline 450 & $150 \pm 30$ & $247 \pm 44$ & $13.5 \pm 4.1$ & $0.057 \pm 0.023$ & $11.9 \pm 3.5$ & $0.623 \pm 0.160$ \\
\hline 520 & $127 \pm 14$ & $261 \pm 31$ & $14.9 \pm 4.9$ & $0.060 \pm 0.025$ & $9.4 \pm 3.4$ & $0.496 \pm 0.052$ \\
\hline 600 & $127 \pm 12$ & $259 \pm 37$ & $15.1 \pm 5.6$ & $0.060 \pm 0.027$ & $9.5 \pm 3.6$ & $0.495 \pm 0.052$ \\
\hline
\end{tabular}

* Mean $\pm 1 \mathrm{SD}$.

Table 6. Measurements at different occlusion times in subgroup $C^{*}$

\begin{tabular}{|c|c|c|c|c|c|c|}
\hline Occlusion time (ms) & $\mathrm{VE}(\mathrm{mL})$ & $\begin{array}{c}\dot{\mathrm{VE}} \\
(\mathrm{mL} / \mathrm{s})\end{array}$ & $\begin{array}{c}\mathrm{Pao}(\mathrm{cm} \\
\left.\mathrm{H}_{2} \mathrm{O}\right) \\
\end{array}$ & $\begin{array}{c}\operatorname{Rrs}\left(\mathrm{cm} \mathrm{H} ~_{2} \mathrm{O} /\right. \\
\mathrm{mL} / \mathrm{s})\end{array}$ & $\begin{array}{c}\mathrm{Crs}(\mathrm{mL} / \mathrm{cm} \\
\left.\mathrm{H}_{2} \mathrm{O}\right)\end{array}$ & $\operatorname{Trs}(\mathrm{s})$ \\
\hline 90 & $215 \pm 36$ & $217 \pm 78$ & $4.6 \pm 1.3$ & $0.023 \pm 0.009$ & $51.2 \pm 19.1$ & $1.097 \pm 0.471$ \\
\hline 140 & $209 \pm 34$ & $233 \pm 74$ & $7.3 \pm 1.1$ & $0.034 \pm 0.010$ & $29.3 \pm 6.1$ & $0.967 \pm 0.321$ \\
\hline 215 & $202 \pm 29$ & $248 \pm 80$ & $8.7 \pm 1.4$ & $0.038 \pm 0.011$ & $24.0 \pm 6.0$ & $0.885 \pm 0.270$ \\
\hline 275 & $201 \pm 29$ & $246 \pm 85$ & $9.2 \pm 1.6$ & $0.041 \pm 0.012$ & $22.3 \pm 4.8$ & $0.897 \pm 0.321$ \\
\hline 320 & $200 \pm 24$ & $258 \pm 86$ & $9.6 \pm 1.6$ & $0.041 \pm 0.012$ & $21.3 \pm 4.3$ & $0.857 \pm 0.299$ \\
\hline 380 & $201 \pm 28$ & $253 \pm 79$ & $10.0 \pm 1.6$ & $0.043 \pm 0.012$ & $20.6 \pm 4.5$ & $0.730 \pm 0.312$ \\
\hline 450 & $194 \pm 22$ & $262 \pm 78$ & $10.3 \pm 1.4$ & $0.044 \pm 0.013$ & $19.2 \pm 3.4$ & $0.817 \pm 0.251$ \\
\hline 520 & $189 \pm 30$ & $259 \pm 98$ & $10.6 \pm 1.5$ & $0.045 \pm 0.014$ & $18.2 \pm 4.2$ & $0.813 \pm 0.277$ \\
\hline 600 & $197 \pm 42$ & $216 \pm 69$ & $10.3 \pm 2.1$ & $0.051 \pm 0.014$ & $19.8 \pm 5.7$ & $0.955 \pm 0.274$ \\
\hline
\end{tabular}

$*$ Mean $\pm 1 \mathrm{SD}$.

time to the next were calculated; changes less than $5 \%$ were considered not significant.

\section{RESULTS}

Figure 1 shows a representative series of tracings obtained in a boy, aged $46 \mathrm{wk}$, with bronchiolitis. As in this example, a straight linear part of the VE-VE plot could be easily obtained in all cases. Table 2 shows VE, VE, Rao, Rrs, Crs, and Trs of the entire group for each occlusion time. For Pao, Rrs, and Crs, the difference between measurements at 90 and $140 \mathrm{~ms}$ was highly significant $(p<0.001)$. In addition, Pao measurements at 140 and $215 \mathrm{~ms}$ differed significantly $(p<0.02)$. Increasing the duration of airway occlusions stepwise from 90 to $320 \mathrm{~ms}$ resulted in marked alterations of measured values from one step to the next; no further relevant changes, however, were observed over the occlusion time range of 320 to $450 \mathrm{~ms}$. Intraindividual variability of the measurements is shown in Table 3; with the exception of measurements after occluding for $90 \mathrm{~ms}$, mean coefficients of variation remained under $10 \%$ and reached a minimum between 320 and $450 \mathrm{~ms}$.

The mean difference of Pao, Rrs, and Crs for each pair of subsequent occlusion times decreased progressively with increasing duration of the occlusions and became minimal above 275 ms. As shown in Figure 2, percentage change of Pao and Rrs fell below the $5 \%$ limit around 275 to $320 \mathrm{~ms}$ and remained there for each pair of the longer occlusions. Percentage change of $\mathrm{Crs}$ decreased continuously toward the $5 \%$ limit from 320 to 380 $\mathrm{ms}$; for the following pairs of occlusion times, changes remained between 5 and $6 \%$.

Findings for each subgroup differed neither from those for the entire group nor from each other (Tables 4-6). Thus, there was no age-related factor in the above-described interrelation of measured values and the duration of the occlusions.

From the occlusion times-Pao plot, the time needed to reach $95 \%$ of maximum $\mathrm{Pao}$ was calculated. This time ranged from 245 to $550 \mathrm{~ms}$ (mean $=386, \mathrm{SD}=67$ ); it did not differ significantly between subgroups, In an attempt to investigate whether pressure equilibration depended on disease severity, this time was correlated to respiratory rate; this correlation did not reach statistical significant for any of the subgroups or for the entire group of children.

\section{DISCUSSION}

This study shows a clear-cut influence of the duration of airway occlusion on the "single-breath" measurement of Rrs and Crs in infants and toddlers with bronchiolitis. The magnitude of this influence, however, is different for different occlusion time ranges. While the measured values changed significantly from 
one occlusion time to the next in the range of the shortest occlusions, the occlusion-time-measurement interrelation curve showed a flat portion between 275 and $450 \mathrm{~ms}$. This finding indicates that occlusion times between 300 and $450 \mathrm{~ms}$ might be ideal for obtaining the degree of respiratory muscle relaxation and pressure equilibration that is required for obtaining reproducible measurements of Rrs and Crs in infants with bronchiolitis.

To a certain extent, the findings of this study are phenomenologic, and their physiologic background remains open for discussion. We speculate that end-inspiratory airway occlusions with a duration of less than $300 \mathrm{~ms}$ may frequently fail to activate the Hering-Breuer reflex. In addition, redistribution of air within the lungs immediately after the occlusion (pendelluft) may have an influence on the time needed to develop pressure equilibration. Furthermore, stress recovery could produce an increase in airway pressure after the occlusion, thereby also having some effect on pressure equilibration time. On the other hand, measurements using occlusions longer than $450 \mathrm{~ms}$ might frequently be hampered by recommencing respiratory muscle activity. All these physiologic speculations should be evaluated by future work, probably using electromyogram recordings of the respiratory muscles.

Theoretically, accurate and reproducible measurements could be obtained by releasing the airway occlusion when observing a plateau in the Pao time curve. This approach would have the advantage of taking into account interindividual variations in the development of a pressure plateau. In preliminary trials, however, our attempts to apply this "plateau-determined" approach met several practical difficulties. The most simple technique, i.e. visually observing the time-based pressure recording and releasing the manual occlusion with the impression of a plateau, results in plateaus of highly variable duration. Too-long occlusions are then frequently invalidated by recommencing respiratory muscle activity. On the other hand, any computerassisted method based on a zero change of Pao would clearly be hampered by minor alterations in Pao, which can frequently be observed when scrutinizing the tracing of a "visually acceptable" plateau. Thus, any plateau-based approach to a timely release of the occlusion is left with the puzzling question of how to define a "real" pressure plateau. As an alternative to struggling with this problem, we investigated the effects of a series of different fixed occlusion times on the measurements obtained and arrived at a clear-cut result, i.e. almost no variability in an occlusion time range from 300 to $450 \mathrm{~ms}$.

These findings were obtained in infants and toddlers with acute viral bronchiolitis, and caution is needed in extrapolating the results to infants without obstructive airway disease. Respiratory drive will be altered in sick infants, and this may have some influence on the duration of occlusion-induced respiratory muscle relaxation. Furthermore, a disease-related reduction of compliance may shorten the time needed for pressure equilibration against the shutter.

The present study could not document an influence of age on the observed interrelation of occlusion times and measurements of Rrs and Crs; passive respiratory mechanics were assessed without difficulties in children up to the age of $1.5 \mathrm{y}$. So far, this method was only applied in newborns and young infants (3-5, 7). The present study suggests that an end-inspiratory airway occlusion might induce respiratory muscle relaxation against the shutter as reliably in the 2 nd as in the 1 st year of life. This finding extends the application of the method significantly beyond the initially investigated age group; in fact, an age-dependent decrease in the efficacy of sedation in toddlers might become a limiting factor for the application of this method rather than any age-dependent decrease in the activity of the Hering-Breuer reflex. This speculation is supported by the results of another recent study that investigated the persistence of the HeringBreuer reflex beyond the neonatal period (8).

In conclusion, the results of the present study indicate that optimum end-inspiratory occlusion time for this single-breath measurement of passive respiratory mechanics in infants and toddlers with bronchiolitis lies in the range of 300 to $450 \mathrm{~ms}$. In addition, the present study demonstrates that the application of this method can be extended far beyond the first months of life.

\section{REFERENCES}

1. Comroe JH, Nisell Ol, Nims RG 1954 A simple method for concurrent measurement of compliance and resistance to breathing in anesthetized animals and man. J Appl Physiol 7:225-228

2. McIlroy MB, Tierney DF, Nadel JA 1963 A new method for measurement of compliance and resistance of lungs and thorax. J Appl Physiol 18:424-427

3. Mortola JP, Fisher JT, Smith B, Fox G, Weeks S 1982 Dynamics of breathing in infants. J Appl Physiol 52:1209-1215

4. LeSouef PN, England SJ, Bryan AC 1984 Passive respiratory mechanics in newborns and children. Am Rev Respir Dis 129:552-556

5. LeSouef PN, England SJ, Bryan AC 1984 Total resistance of the respiratory system in preterm infants with and without an endotracheal tube. $J$ Pediatr 104:108-111

6. Bergman NA 1966 Measurement of respiratory resistance in anesthetized subjects. J Appl Physiol 21:1913-1917

7. Guslits BG, Wilkie RA, England SJ, Bryan AC 1987 Comparison of methods of measurement of compliance of the respiratory system in children. Am Rev Respir Dis 136:727-729

8. Rabbette PS, Costeloe KL, Stocks J 1991 Persistence of the Hering-Breuer reflex beyond the nconatal period. J Appl Physiol 71:474-480 